\title{
Approximation of a Class of Wiener Integrals*
}

\author{
By Lloyd D. Fosdick
}

1. Introduction. Many physical problems can be formulated in terms of the conditional Wiener integral of the functional [1], [2]

$$
F(x)=\exp \left[-\int_{0}^{1} V(x(\tau)) d \tau\right]
$$

and since evaluation of this integral by analysis often cannot be done, it is of some practical interest to investigate the possibility of numerically evaluating this integral. Such a calculation is expected to require an enormous amount of arithmetic since it involves the evaluation of a $k$-fold integral where $k \gg 1$; an exact evaluation requires going to the limit $k \rightarrow \infty$. Consequently, it is taken for granted that the services of a large computing machine may be required.

In this paper we derive an approximation formula for the conditional Wiener integral of the functional $F(x)$ in Eq. (1.1) that may be useful for machine calculations. The main result is the formula

$$
E\{F(x) \mid x(1)=X\}=A \int_{-\infty}^{+\infty} \int_{-\infty}^{+\infty} \cdots \int_{-\infty}^{+\infty} F_{k} g_{k} \omega_{k} d x_{1} d x_{2} \cdots d x_{k-1}+\Delta_{k}
$$

where $E\{F(x) \mid x(1)=X\}$ stands for the Wiener integral of the functional $F(x)$ with the end point conditions $x(0)=0, x(1)=X ; F_{k}$ is the functional $F(x)$ when $x(\tau)$ is a piecewise straight line with breaks at $0<\tau_{1}<\tau_{2}<\cdots<\tau_{k-1}<1$, and $\tau_{i+1}-\tau_{i}=1 / k$ for all $i ; g_{k}$ is given by

$$
g_{k}=\prod_{i=1}^{k}\left(1-\frac{1}{2 k^{2}} \int_{0}^{1} V^{\prime \prime}\left(x_{i-1}+\left(x_{i}-x_{i-1}\right) \tau\right) \tau(1-\tau) d \tau\right),
$$

where $x_{i}=x\left(\tau_{i}\right), x_{0}=0, x_{k}=X$, and $V^{\prime \prime}(y)=d^{2} V / d y^{2} ; \omega_{k}$ is the Gaussian weight factor

$$
\omega_{k}=\prod_{i=1}^{k}\left(\frac{k}{2 \pi}\right)^{1 / 2} \exp \left(-\frac{k\left(x_{i}-x_{i-1}\right)^{2}}{2}\right)
$$

$A$ is the normalization factor

$$
A=\sqrt{ }(2 \pi) \exp \left(\frac{X^{2}}{2}\right)
$$

and, finally, $\Delta_{k}$, which is the error when the first term on the right side of Eq. (1.2) is used as an appoximation for the Wiener integral, is bounded above and below by an expression which is $O\left(1 / k^{2}\right)$. A related result with an error term $O\left(1 / k^{8}\right)$ is also given.

Received March 16, 1964.

* Most of this work was supported by the AEC and was performed at the Applied Mathematics Division of the Argonne National Laboratory; a portion of it was done at the MaxPlanck-Institut für Physik und Astrophysik with the support of a fellowship from the John Simon Guggenheim Memorial Foundation. 
A simple way to describe the derivation of the above results is by analogy with a well-known technique for the Riemann integral. In these terms our approach is simply to subdivide the interval of integration and expand the integranc in a Taylor series in each subdivision. Truncation of the Taylor series and integration of the resultant polynomial yields the approximation, and consideration of the remainder term yields an estimate of the error.

Our second-order formula seems to be simpler than Cameron's "Simpson's Rule" [3] which has the same accuracy, $O\left(1 / k^{2}\right)$, hence it may be more suitable for actual machine calculation. In any case, since our approach is different from Cameron's it may be of some interest to others concerned with developing approximation schemes for Wiener integrals. Finally, we wish to point out that the technique used here can be continued in an obvious way to obtain more accurate formulas.

2. Preliminary Remarks on the Conditional Wiener Integral. The condi:ional Wiener integral $E\{F(x) \mid x(1)=X\}$ may be defined as follows [1]:

$$
E\{F(x) \mid x(1)=X\}=\lim _{n \rightarrow \infty} A \int_{-\infty}^{+\infty} \int_{-\infty}^{+\infty} \cdots \int_{-\infty}^{\infty} F_{n} \omega_{n} d x_{1} d x_{2} \cdots d x_{n-1},
$$

where $A, F_{n}$, and $\omega_{n}$ are the same as in Eq. (1.2) with $n$ replacing $k$. We will assume that $V(x)$ is continuous and bounded from below; these conditions are sufficient to insure the existence of the limit in Eq. (2.1). Later we will assume the existence and continuity of certain derivatives of $V(x)$.

The manner in which the $\tau$-interval is partitioned in passing to the limit in Eq. (2.1) is arbitrary. To establish our approximation formulas it is partitioned so that $k-1$ points $\tau_{1}, \tau_{2}, \cdots, \tau_{k-1}$ are fixed and we make use of the formula

$$
E\{F(x) \mid x(1)=X\}=A \int_{-\infty}^{+\infty} \int_{-\infty}^{+\infty} \cdots \int_{-\infty}^{+\infty} I_{k} \omega_{k} d x_{1} d x_{2} \cdots d x_{k-1},
$$

where

$$
I_{k}=\lim _{n \rightarrow \infty} \omega_{k}^{-1} \int_{-\infty}^{+\infty} \int_{-\infty}^{+\infty} \cdots \int_{-\infty}^{+\infty} F_{n} \omega_{n} d x_{k+1} d x_{k+2} \cdots d x_{n-1}
$$

and

$$
x_{k}=x_{n}=X .
$$

For definiteness we can assume that this partitioning is realized by repeated halving (i.e., $\tau_{1}=1 / 2, \tau_{2}=1 / 4, \tau_{3}=3 / 4, \tau_{4}=1 / 8, \cdots$ ); then, for a proper interpretation of $\omega_{k}$, Eq. (1.4), it is necessary to relabel the points so that for neighboring points $\tau_{i}, \tau_{j}$ with $\tau_{i}<\tau_{j}$, the relation $j=i+1$ is satisfied.

Eq. (2.2) is referred to as the mixed integration formula. This formula is used to obtain a family of approximations to $E\{F(x) \mid x(1)=X\}$ from approximations for $I_{k}$. The latter are formed by expansion of the functional in a generalized Taylor series about the piecewise straight line broken at the points $0<\tau_{1}<\tau_{2}<\cdots$ $<\tau_{k-1}<1$. This is particularly convenient because of the way the functional can be factored. Since the Wiener integral for any term in this series may be evaluated analytically we are thus led to an approximation for $I_{k}$ when the series is truncated. 
This is treated in detail in the next sections.

Though it is aside from our main concern here, a remark about the evaluation of the $(k-1)$-fold integral on the right side of Eq. (2.2) may be of interest. Let us suppose that an approximation for $I_{k}$ has been obtained; in general, it will depend on $x_{1}, x_{2}, \cdots, x_{k-1}$ and the $(k-1)$-fold quadrature in Eq. (2.2) will have to be performed numerically. If this is done by Monte Carlo sampling it is worth noting that the constraint at the right endpoint (i.e., $x(1)=X$ ) causes no special difficulty. Monte Carlo sampling would consist in selecting $(k-1)$-tuples $\left\{x_{1}, x_{2}, \cdots, x_{k-1}\right\}$ according to the probability $\omega_{k}$, where $x_{0}=0$ and $x_{k}=X$. The elements $x_{1}, x_{2}, \cdots, x_{k-1}$ can be picked one at a time by repeated application of the so-called "interpolation formula" [4]: Let $x_{a}$ and $x_{c}, \tau_{a}<\tau_{c}$ be given, then $x_{b}$ for $\tau_{a}<\tau_{b}<\tau_{c}$ may be represented by

$$
x_{b}=\frac{x_{a}\left(\tau_{c}-\tau_{b}\right)+x_{c}\left(\tau_{b}-\tau_{a}\right)}{\tau_{c}-\tau_{a}}+\xi \sqrt{ }\left(\frac{\left(\tau_{b}-\tau_{a}\right)\left(\tau_{c}-\tau_{b}\right)}{\left(\tau_{c}-\tau_{a}\right)}\right),
$$

where $\xi$ is a reduced Gaussian random variable. ${ }^{1}$ Hence, with the help of a generator for Gaussian random variables, the $(k-1)$-tuples can be selected in a straightforward way.

3. The Generalized Taylor Series. The important material in this section is taken from a paper by Graves [5]. We begin with the definition of the $n$th variation of the functional $F(x)$. The space of continuous functions $x(\tau)$ is denoted by $C$.

Definition. We say that $F(x)$ has an $n$th variation at $x_{0} \in C$ in case for every $\delta x \in C$ the function of the real variable $r, F\left(x_{0}+r \delta x\right)$, has an $n$th derivative at $r=0$. This $n$th derivative at $r=0$ is denoted by $\delta^{n} F\left(x_{0}, \delta x\right)$; we denote the $n$th derivative at $r$ by $\delta^{n} F\left(x_{0}+r \delta x, \delta x\right)$.

Let us consider an example related to our own application. Suppose

$$
F(x)=\exp \left(-\int_{0}^{1} x^{4}(\tau) d \tau\right)
$$

then the first two variations at $x_{0}$ are

$$
\delta^{1} F\left(x_{0}, \delta x\right)=-4\left(\int_{0}^{1} x_{0}^{3}(\tau) \delta x d \tau\right) F\left(x_{0}\right)
$$

and

$$
\delta^{2} F\left(x_{0}, \delta x\right)=16\left(\int_{0}^{1} x_{0}{ }^{3}(\tau) \delta x d \tau\right)^{2} F\left(x_{0}\right)-12\left(\int_{0}^{1} x_{0}{ }^{2}(\tau)(\delta x)^{2} d \tau\right) F\left(x_{0}\right)
$$

The generalized Taylor's Theorem, in a more restricted form than in Graves' paper, is:

THeorem. Suppose that $F(x)$ has an nth variation at every $x \in C$. Suppose that for every $x_{1}, x_{2} \in C$, the function of the real variable $r, \delta^{n} F\left(x_{1}+\left(x_{2}-x_{1}\right) r, x_{2}-x_{1}\right)$, is bounded on the interval $(0,1)$ and its set of discontinuities has measure zero. Then, for every $x_{1}, x_{2} \in C$, we have

$$
F\left(x_{2}\right)=F\left(x_{1}\right)+\sum_{i=1}^{n-1} \delta^{i} F\left(x_{1}, x_{2}-x_{1}\right) / i !+R_{n}\left(x_{1}, x_{2}\right),
$$

$1 \bar{\xi}=0 . \bar{\xi}^{2}=1$. 
where

$$
R_{n}\left(x_{1}, x_{2}\right)=\int_{0}^{1} \delta^{n} F\left(x_{1}+\left(x_{2}-x_{1}\right) r, x_{2}-x_{1}\right) \frac{(1-r)^{n-1}}{(n-1) !}
$$

Once again let us suppose that $F(x)$ is given by Eq. (3.1), then with this theorem and Eqs. (3.2), (3.3), we have, for example,

$$
\begin{array}{r}
F\left(x_{1}+\delta x\right)=F\left(x_{1}\right)\left(1-4 \int_{0}^{1} x_{1}^{3} \delta x d \tau+8\left(\int_{0}^{1} x_{1}^{3} \delta x d \tau\right)^{2}-6 \int_{0}^{1} x_{1}{ }^{2}(\delta x)^{2} d \tau\right) \\
+R_{3}\left(x_{1}, x_{1}+\delta x\right)
\end{array}
$$

where

$$
R_{3}\left(x_{1}, x_{1}+\delta x\right)=\int_{0}^{1} \delta^{3} F\left(x_{1}+r \delta x, \delta x\right) \frac{(1-r)^{2}}{2} d r
$$

and

$$
\begin{aligned}
\delta^{3} F\left(x_{1}+r \delta x, \delta x\right)= & F\left(x_{1}+r \delta x\right)\left(-64\left(\int_{0}^{1}\left(x_{1}+r \delta x\right)^{3} \delta x d \tau\right)^{3}\right. \\
& +144\left(\int_{0}^{1}\left(x_{1}+r \delta x\right)^{3} \delta x d \tau\right)\left(\int_{0}^{1}\left(x_{1}+r \delta x\right)^{2}(\delta x)^{2} d \tau\right) \\
& \left.-24 \int_{0}^{1}\left(x_{1}+r \delta x\right)(\delta x)^{3} d \tau\right) .
\end{aligned}
$$

It is, of course, to be understood that here $x_{1}$ stands for a particular function of $\tau$.

4. Normal Form of the Conditional Wiener Integral. In the next section we will make use of the fact that the conditional Wiener integral is invariant with respect to the change of variables $x(\tau) \rightarrow y(\tau)$, where

$$
y(\tau)=x(\tau)-(\alpha \tau+\beta),
$$

and $\alpha$ and $\beta$ are arbitrary constants. Specifically, this means that

$$
\begin{aligned}
E\{F(x(\tau)) \mid x(1) & =X\} \\
& =E\{F(y(\tau)+\alpha \tau+\beta) \mid y(0)=-\beta, y(1)=X-\alpha-\beta\},
\end{aligned}
$$

where, on the right side, $y(\tau)$ is regarded as the variable of integration. Eq. (4.2) is easily obtained from the following considerations. In the Gaussian weight factor

$$
\omega_{n}(x)=\prod_{i=1}^{n}\left(\frac{n}{2 \pi}\right)^{1 / 2} \exp \left(\frac{-n\left(x_{i}-x_{i-1}\right)^{2}}{2}\right),
$$

replace $x_{i}$ by $y_{i}+\alpha \tau_{i}+\beta$ for $i=0,1, \cdots, n$, then this becomes

$$
\omega_{n}(y+\alpha \tau+\beta)=\left(\prod_{i=1}^{n}\left(\frac{n}{2 \pi}\right)^{1 / 2} \exp \left(-\frac{n\left(y_{i}-y_{i-1}\right)^{2}}{2}\right)\right) \exp \left(-\alpha X+\frac{\alpha^{2}}{2}\right),
$$

or

$$
\omega_{n}(y+\alpha \tau+\beta)=\omega_{n}(y) \exp \left(-\alpha X+\frac{\alpha^{2}}{2}\right) .
$$


The Jacobian of the transformation $x \rightarrow y$ is unity, hence

$$
\begin{gathered}
\sqrt{ }(2 \pi) \exp \left(\frac{X^{2}}{2}\right) \int_{-\infty}^{+\infty} \int_{-\infty}^{+\infty} \cdots \int_{-\infty}^{+\infty} F_{n}(x) \omega_{n}(x) d x_{1} d x_{2} \cdots d x_{n-1} \\
=\sqrt{ }(2 \pi) \exp \left(\frac{(X-\alpha)^{2}}{2}\right) \int_{-\infty}^{+\infty} \int_{-\infty}^{+\infty} \\
\cdots \int_{-\infty}^{+\infty} F_{n}(y+\alpha \tau+\beta) \omega_{n}(y) d y_{1} d y_{2} \cdots d y_{n-1}
\end{gathered}
$$

for all $n$ and therefore in the limit as $n \rightarrow \infty$, thus giving the relation cited in Eq. (4.2). It is to be noted that this result means that a given Wiener integral subject to arbitrary endpoint conditions is equal to one with endpoint conditions $x(0)=0$ and $x(1)=0$ when the variable of integration is changed according to Eq. (4.1). When the endpoint conditions are $x(0)=0$ and $x(1)=0$ we will say that the integral is expressed in normal form.

Another useful property that is easily verified is that the change in variables

$$
\tau \rightarrow k\left(\tau+\tau_{0}\right), \quad y(\tau) \rightarrow \sqrt{ } k y(\tau)
$$

leaves the form of the Wiener integral unchanged. This means that the origin of the $\tau$-interval is arbitrary and the length of this interval can be changed by making a corresponding change in the magnitude of $y(\tau)$.

5. The Approximation for $I_{k}$. The functional in Eq. (1.1) can be factored:

$$
F(x)=\prod_{i=1}^{k} f_{i}(x),
$$

where

$$
f_{i}(x)=\exp \left(-\int_{\tau_{i-1}}^{\tau_{i}} V(x(\tau)) d \tau\right) .
$$

It follows that $I_{k}$, Eq. (2.3), can be expressed as a product of conditional Wiener integrals

$$
I_{k}=\prod_{i=1}^{k} E\left\{f_{i}(x) \mid x\left(\tau_{i-1}\right)=x_{i-1}, x\left(\tau_{i}\right)=x_{i}\right\},
$$

where the $\tau$-interval for the $i$ th factor is $\tau_{i}-\tau_{i-1}$.

Let us now fix attention on an arbitrary factor in this product and for convenience introduce new variables

$$
\begin{aligned}
\eta & =k\left(\tau-\tau_{i-1}\right), \\
y(\eta) & =\sqrt{ } k\left(x(\tau)-\left(x_{i}-x_{i-1}\right) \eta-x_{i-1}\right),
\end{aligned}
$$

which put the factor in normal form; i.e., we will consider the factor

where

$$
E\left\{f_{i}(y) \mid y(1)=0\right\},
$$

$$
f_{i}(y)=\exp \left(-\frac{1}{k} \int_{0}^{1} V\left(\frac{y(\eta)}{\sqrt{ } k}+s_{i}(\eta)\right) d \eta\right)
$$


and

$$
s_{i}(\eta)=\left(x_{i}-x_{i-1}\right) \eta+x_{i-1} .
$$

We will assume that the conditions of the generalized Taylor Theorem are satisfied for $n=4$, and that

$$
\frac{d^{4}}{d r^{4}} V\left(r y(\eta)+s_{i}(\eta)\right)
$$

is a uniformly continuous function of $\eta$ for all $x_{i}$. It should be remarked that $y(\eta)$ is a uniformly continuous function of $\eta$ because of the fact that the Wiener measure is entirely concentrated on the continuous functions $y(\eta)$ which satisfy the Lipschitz condition

$$
|y(\eta+h)-y(\eta)|<c \sqrt{\left(2 h \log \frac{1}{h}\right)}
$$

for all $0 \leqq \eta \leqq 1-h$ and for $h<H(c)$ where $c>1$ [4]. The uniform continuity condition is imposed so that the order of differentiation with respect to $r$ and integration with respect to $\eta$ may be interchanged. Expressing $f_{i}(y)$ as a Taylor series about $y=0$, one finds

$$
\begin{aligned}
f_{i}(y)=f_{i}(0)\left(1-\frac{1}{k^{3 / 2}} \int_{0}^{1} V^{\prime}\left(s_{i}\right) y d \eta\right. & \\
+ & \frac{1}{2}\left(\frac{1}{k^{3}}\left(\int_{0}^{1} V^{\prime}\left(s_{i}\right) y d \eta\right)^{2}-\frac{1}{k} \int_{0}^{1} V^{\prime \prime}\left(s_{i}\right) y^{2} d \eta\right) \\
+ & \frac{1}{6}\left(-\frac{1}{k^{9 / 2}}\left(\int_{0}^{1} V^{\prime}\left(s_{i}\right) y d \eta\right)\right) \\
& +\frac{3}{k^{7 / 2}}\left(\int_{0}^{1} V^{\prime}\left(s_{i}\right) y d \eta\right) \cdot\left(\int_{0}^{1} V^{\prime \prime}\left(s_{i}\right) y^{2} d \eta\right) \\
& \left.-\frac{1}{k^{5 / 2}} \int_{0}^{1} V^{\prime \prime \prime}\left(s_{i}\right) y^{3} d \eta\right)+R_{4}(0, y),
\end{aligned}
$$

where $s_{i}(\eta) \equiv s_{i}$ and all terms up to the third variation of the functional are explicit. Now we will neglect the remainder term, $R_{4}(0, y)$, and use the resulting approximation $f_{i}{ }^{*}(y)$ for $f_{i}(y)$ as the integrand in the Wiener integral; i.e., we will calculate $E\left\{f_{i}^{*}(y) \mid y(1)=0\right\}$, where

$$
f_{i}^{*}(y)=f_{i}(y)-R_{4}(0, y) .
$$

This calculation is easily made after the order of integration is changed and the Wiener integration is executed first; the result is

$E\left\{f_{i}^{*}(y) \mid y(1)=0\right\}$

$$
\begin{aligned}
=f_{i}(0)\left(1+\frac{1}{k^{3}} \int_{0}^{1} \int_{0}^{\eta_{2}} V^{\prime}\left(s_{i}\left(\eta_{1}\right)\right)\right. & V^{\prime}\left(s_{i}\left(\eta_{2}\right)\right) \cdot \eta_{1}\left(1-\eta_{2}\right) d \eta_{1} d \eta_{2} \\
& \left.-\frac{1}{2 k^{2}} \int_{0}^{1} V^{\prime \prime}\left(s_{i}(\eta)\right) \eta(1-\eta) d \eta\right) .
\end{aligned}
$$


To arrive at this result we have used the well-known relation ${ }^{2}$

$$
E\left\{y\left(\eta_{1}\right) y\left(\eta_{2}\right) \mid y(1)=0\right\}=\eta_{1}\left(1-\eta_{2}\right) \quad\left(\eta_{1} \leqq \eta_{2}\right)
$$

and the fact that the integral of a product of $y(\eta)$ 's containing an odd number of factors is zero, specifically,

$$
\begin{array}{r}
E\{y(\eta) \mid y(1)=0\}=0, \\
E\left\{y\left(\eta_{1}\right) y\left(\eta_{2}\right) y\left(\eta_{3}\right) \mid y(1)=0\right\}=0 .
\end{array}
$$

The interchange of the order of integration is justified as follows. First of all, the integrals on the right side of (5.9) exist by virtue of our initial assumptions. Now if $C_{y}$ is the space of continuous functions $y(\eta)(y(0)=0, y(1)=0)$ and $T$ is the interval $(0,1)$ on the real line, the functions $y(\eta), y\left(\eta_{1}\right) y\left(\eta_{2}\right)$ are integrable in the product spaces $C_{y} \times T, C_{y} \times T \times T$, etc., and therefore so are $V^{\prime}\left(s_{i}\right) y(\eta)$, $V^{\prime}\left(s_{i}\left(\eta_{1}\right)\right) V^{\prime}\left(s_{i}\left(\eta_{2}\right)\right) y\left(\eta_{1}\right) y\left(\eta_{2}\right)$, etc. It then follows from Fubini's theorem that the order of integration can be changed.

In Eq. (5.11) we drop the term of higher order in $1 / k$ to obtain

$$
\left[E\left\{f_{i}^{*}(y) \mid y(1)=0\right\}\right]^{*}=f_{i}(0)\left(1-\frac{1}{2 k^{2}} \int_{0}^{1} V^{\prime \prime}\left(s_{i}\right) \eta(1-\eta) d \eta\right) .
$$

This is taken as the basic approximation for $E\left\{f_{i}(y) \mid y(1)=0\right\}$ and when it is used in Eq. (5.3) it provides an approximation for $I_{k}$ which then, with the help of Eq. (2.2), yields an approximation for $E\{F(x) \mid x(1)=X\}$.

The latter approximation is just the first term on the right side of Eq. (1.2). Now we want to examine the nature of the second term $\Delta_{k}$ on the right side of Eq. (1.2) which represents the error; it will be shown that, for large $k$,

$$
\Delta_{k} \sim O\left(\frac{1}{k^{2}}\right)
$$

From Eqs. (5.10), (5.11), and (5.15),

$$
\begin{aligned}
E\left\{f_{i}(y) \mid y(1)=\right. & 0\}-\left[E\left\{f_{i}^{*}(y) \mid y(1)=0\right\}\right]^{*} \\
= & E\left\{R_{4}(0, y) \mid y(1)=0\right\} \\
& +\frac{f_{i}(0)}{k^{3}} \int_{0}^{1} \int_{0}^{\eta_{2}} V^{\prime}\left(s_{i}\left(\eta_{1}\right)\right) V^{\prime}\left(s_{i}\left(\eta_{2}\right)\right) \eta_{1}\left(1-\eta_{2}\right) d \eta_{1} d \eta_{2} .
\end{aligned}
$$

The remainder $R_{4}(0, y)$ contains the fourth variation, $\delta^{4} f_{i}(r y, y)$, of the functional and a short calculation shows

$$
\delta^{4} f_{i}(r y, y)=-\frac{f_{i}(r y)}{k^{3}} \int_{0}^{1} V^{(\mathrm{IV})}\left(\frac{r y(\eta)}{\sqrt{ } k}+s_{i}(\eta)\right) y^{4}(\eta) d \eta+O\left(\leqq \frac{1}{k^{4}}\right)
$$

Hence the error in the basic approximation, Eq. (5.15), may be written

$$
\begin{aligned}
\epsilon_{i} & =E\left\{f_{i}(y) \mid y(1)=0\right\}-\left[E\left\{f_{i}^{*}(y) \mid y(1)=0\right\}\right]^{*} \\
& =\frac{\phi_{i}}{k^{3}},
\end{aligned}
$$

${ }^{2}$ Easily derived from Eq. (2.4). 
where $\phi_{i}$, as a function of $k$, is bounded. The approximation used for $I_{k}$ is

$$
\begin{aligned}
I_{k}^{*} & =\prod_{i=1}^{k}\left[E\left\{f_{i}^{*}(y) \mid y(1)=0\right\}\right]^{*} \\
& =I_{k} \prod_{i=1}^{k}\left[1-\frac{\phi_{i}}{k^{3} E\left\{f_{i}(y) \mid y(1)=0\right\}}\right]
\end{aligned}
$$

hence

$$
I_{k}-I_{k}^{*}=I_{k} \psi_{i}(k),
$$

where $\psi_{i}(k)=O\left(1 / k^{2}\right)$. Finally, $\Delta_{k}$ is given by

$$
\Delta_{k}=A \cdot \int_{-\infty}^{+\infty} \int_{-\infty}^{+\infty} \cdots \int_{-\infty}^{+\infty} I_{k} \psi_{i}(k) \omega_{k} d x_{1} d x_{2} \cdots d x_{k-1}
$$

which is also $O\left(1 / k^{2}\right)$ because of the character of $\psi_{i}(k)$.

In an entirely analogous way higher-order approximations may be derived. For example, corresponding to the basic approximation (5.15), one can derive the following approximation for $E\left\{f_{i}(y) \mid y(1)=0\right\}$ :

$$
\begin{aligned}
E\left\{f_{i}^{*}(y) \mid y(1)=0\right\}=f_{i}(0) & \left(1-\frac{1}{2 k^{2}} \int_{0}^{1} V^{\prime \prime}\left(s_{i}\right) \eta(1-\eta) d \eta\right. \\
+ & \frac{1}{k^{3}} \int_{0}^{1} \int_{0}^{\eta_{2}} V_{1}^{\prime}\left(s_{i}\right) V_{2}^{\prime}\left(s_{i}\right) \eta_{1}\left(1-\eta_{2}\right) d \eta_{1} d \eta_{2} \\
& \left.\quad-\frac{3}{4 ! k^{3}} \int_{0}^{1} V^{(\mathrm{IV})}\left(s_{i}\right) \eta^{2}(1-\eta)^{2} d \eta\right) .
\end{aligned}
$$

The error in the approximation (5.25) is $O\left(1 / k^{4}\right)$, and it leads then to a total error in the approximation for $E\{F(x) \mid x(1)=X\}$ (analogous to $\Delta_{k}$ above) which is $O\left(1 / k^{3}\right)$.

6. Practical Considerations. We conclude with two brief remarks about the application of the above formulas in a machine calculation. It was pointed out earlier, in Section 2, that $x_{1}, x_{2}, \cdots, x_{k-1}$ can be chosen one at a time in a Monte Carlo calculation. With each choice it is possible to calculate the factor $E\left\{f_{i}^{*}(y) \mid y(1)=0\right\}$ (or the corresponding higher approximations), and so one can arrange the calculation of $I_{k}{ }^{*}$ in a simple way without requiring a vast amount of intermediate storage when $k$ is large.

It would be wasteful of time in most computers to literally calculate each factor $E\left\{f_{i}^{*}(y) \mid y(1)=0\right\}$. A more efficient scheme is to accumulate the sum of the integrals

$$
S_{k}=-\frac{1}{k} \sum_{i=1}^{k} \int_{0}^{1} V\left(s_{i}\right) d \eta
$$

and then form $\exp \left(S_{k}\right)$ to get the contribution from the product of $f_{i}(0)$ 's. This must be multiplied by $\prod_{i=1}^{k}\left(1-\left(1 / 2 k^{2}\right) \int_{0}^{1} V^{\prime \prime}\left(s_{i}\right) \eta(1-\eta) d \eta\right)$, but if we are willing

${ }^{3} V_{j}^{\prime}\left(s_{i}\right) \equiv V^{\prime}\left(s_{i}\left(\eta_{j}\right)\right)$ 
to admit a slightly larger error we can, of course, use the approximation

$$
\prod_{i=1}^{k}\left(1-\frac{a_{i}}{k^{2}}\right) \cong 1-\frac{1}{k^{2}} \sum_{i=1}^{k} a_{i}
$$

without changing the order of the error term; in most computers this approximation should result in a significant saving of time, since multiplication can be expected to take at least twice as much time as addition.

Digital Computer Laboratory, University of Illinois Applied Mathematics Division, Argonne National Laboratory

1. I. M. GEL'FAND \& A. M. JAGLOM, "Integration in function spaces and its application to quantum physics," Uspehi Mat. Nauk, v. 11, 1956, no. 1 (67), pp. 77-114; English transl., J. Mathematical Phys., v. 1, 1960, pp. 48-69. MR 17, 1261; MR 22 \#3455.

2. S. G. Brost, "Functional integrals and statistical physics," Rev. Mod. Phys., v. 33, 1961, pp. 79-92. MR 24 \#B306.

3. R. H. CAMERON, "A 'Simpson's rule' for the numerical evaluation of Wiener's integrals in function space," Duke Math. J., v. 18, 1951, pp. 111-130. MR 12, 718.

4. P. LEvy, Le Mouvement Brownien, Mémor. Sci. Math., Fasc. 126, Gauthier-Villars, Paris, 1954. MR 16, 601.

5. L. M. Graves, "Riemann integration and Taylor's theorem in general analysis," Trans. Amer. Math. Soc., v. 29, 1927, pp. 163-177. 